\title{
Association between systemic lupus erythematosus and cancer: findings from cohort studies and Mendelian randomization analysis
}

Dongqing Gu

Army Medical University

Mingshuang Tang

Army Medical University

Huijie Cui

Sichuan University

Min Zhang

Chongqing Medical University

Yutong Wang

Army Medical University

Ye Bai

Chongqing Medical University

Xin Wang

Sichuan University

Ben Zhang ( $\sim$ benzhang@vip.163.com )

Army Medical University

\section{Research Article}

Keywords: Systemic lupus erythematosus, Cancer, Causal association, Meta-analysis, Mendelian randomization

Posted Date: August 24th, 2021

DOl: https://doi.org/10.21203/rs.3.rs-841744/v1

License: (c) (i) This work is licensed under a Creative Commons Attribution 4.0 International License. Read Full License 


\section{Abstract \\ Background}

Observational studies suggested that systemic lupus erythematosus (SLE) was associated with an increased risk of cancer, however, the causal effect remains unclear. We aim to determine the causality between SLE and cancer using a meta-analysis and Mendelian randomization (MR) approach.

\section{Methods}

A systematic search was conducted using PubMed to identify cohort studies published before January 21,2021 . Meta-analysis was performed to calculate relative risk (RR) and corresponding $95 \%$ confidence intervals $(\mathrm{Cl})$, and the potentially causal relationships identified by observational studies were further validated using two-sample Mendelian randomization.

\section{Results}

Through meta-analysis of 43 cohort studies involving 231,499 patients, we observed an increased overall cancer risk among SLE patients $(R R=1.62,95 \% \mathrm{Cl}, 1.47-1.79)$. Site-specific analysis suggested that SLE patients were associated with an increased risk of 17 cancers. Mendelian randomization analysis indicated that genetically predisposed SLE was causally associated with an increased risk of lymphoma (odds ratio $=1.0004,95 \% \mathrm{Cl}, 1.0001-1.0007, \mathrm{P}=0.0035$ ), whereas a decreased risk of bladder cancer (odds ratio $=0.9996,95 \% \mathrm{Cl}, 0.9994-0.9998, \mathrm{P}=0.00004$ ) in European ancestry. However, no relationship was observed between genetically predisposed SLE and risk of colon, pancreatic, lung, cervical and Nonmelanoma skin cancer in European ancestry, liver cancer and lung cancer in Asian ancestry.

\section{Conclusions}

Findings from meta-analysis and Mendelian randomization analysis suggested that SLE might be causally associated with an increased risk of lymphoma. However, inconsistent results were observed between SLE and risk of bladder cancer.

\section{Introduction}

Systemic lupus erythematosus (SLE) is an autoimmune disease characterized by the presence of nuclear autoantibodies which could cause immune complexes formation, and thus resulting in inflammation of multiple organs ${ }^{1}$. Globally, the prevalence of SLE has been estimated to be $30-150$ per 100,000 , and incidence ranges from 2.2 to 23.1 per 100,000 each year ${ }^{1}$. It is reported that there is approximately 500,000 SLE patients in Europe and 250,000 in the USA ${ }^{2,3}$. The clinical presentation of SLE is 
heterogeneous and can involve one or more organs, including the skin, joints, kidneys, blood cells, and nervous system, taking a chronic or relapsing and remitting disease course ${ }^{4}$.

In past decades, various observational studies have investigated the relationship between SLE and cancers. Patients with SLE generally carry an increased risk of developing cancers. However, the relationship between SLE and cancer is complex. Recent epidemiologic evidences have suggested that there is an increased risk of some malignancies, including lung cancer, liver cancer, cervical cancer, and especially some hematologic cancers, such as non-Hodgkin lymphoma (NHL), Hodgkin lymphoma (HL) and leukemia among patients with SLE ${ }^{5-10}$. To the contrary, some studies have found a decreased risk of some hormone-sensitive cancers, such as breast, ovarian, and endometrial cancer, in SLE patients ${ }^{11-}$ 13. Importantly, the causal role of SLE for cancer is weak since inference from observational studies is limited by residual or unmeasured confounding, and other biases such as reverse causation and detection bias ${ }^{14,15}$.

In recent years, Mendelian randomization (MR) analysis has been widely used to assess potential causal estimates of various risk factors with outcomes of interest. This approach has the advantage over conventional observational studies of minimizing potential biases by using genetic markers as instrumental variables of environmental risk factors ${ }^{16}$. Two-sample Mendelian randomization is an extension of this methodology deriving estimates for the required genotype-exposure and genotypeoutcome associations from separate and nonoverlapping samples of the same representative population 17,18 . This approach exploit the rapidly growing availability of summary data from large consortia of genome-wide association studies (GWASs), and thus allow greater sample sizes, to improve statistical power ${ }^{19}$. Furthermore, in terms of SLE, genetic variants play an important role in SLE occurrence. The heritability of SLE was estimated to range from $8.7-16.0 \%$, suggesting a novel and promising instrumental variable for analyzing the causal relationship between SLE and disease risk ${ }^{20}$. Peng et al. has identified SLE as a causal risk factor for lung cancer using Mendelian randomization analysis ${ }^{21}$. Therefore, the Mendelian randomization analysis may be an ideal approach to evaluate the causality between SLE and specific cancers.

In the present study, we systematically investigate the correlation between SLE and overall cancer and site-specific cancers risk by conducting a meta-analysis of published cohort studies. Furthermore, subgroup meta-analyses by region (Europe, America, or Asia) were performed. Then, utilizing SLE-related SNPs as instrumental variables identified by the GWAS with largest sample size, we investigated the correlation between genetically predisposed SLE and site-specific cancers risk using data from MR-Base. We aim to determine the causality between SLE and site-specific cancers based on findings from metaanalysis of cohort studies and Mendelian randomization analysis. Otherwise, we estimate the causespecific standard mortality ratio (SMR) for cancer in SLE patients. This comprehensive study will provide epidemiological evidence supporting the associations between SLE and cancer risk. These evidences could be utilized to drive public policies and to help guide personalized medicine to better manage SLE and reduce associated cancer morbidity and mortality. 


\section{Materials And Methods}

Our methodology for the meta-analysis followed the guidelines proposed by the Preferred Reporting Items for Systematic Reviews and Meta-Analyses (PRISMA) statement ${ }^{22}$. Review protocol of this meta-analysis was registered in PROSPERO (CRD42021243635).

\section{Meta-analysis of Cohort Studies}

We searched PubMed to identify cohort studies that investigated the association between SLE and cancer risk published before January 21, 2021 (Fig. 1). We combined relative risks (RRs) of overall cancer and sites-specific cancer in SLE patients using a random-effects meta-analysis. For studies not containing RR, standard incidence ratio (SIR), odds ratio (OR) or hazard ratio (HR) were treated as the substitute of RR in the current study. We also performed subgroup meta-analyses based on region (Europe, America, or Asia). We used the Cochran's Q statistic to test for heterogeneity and the ${ }^{2}$ statistic to quantify heterogeneity across studies. Potential publication bias was assessed using Begg's and Egger's approaches. Sensitivity analyses were performed to evaluate the robustness of the results. We considered $P$ values of less than 0.10 in tests of heterogeneity and publication bias, and $P$ values of less than 0.05 in the meta-analyses to be statistically significant. All tests were two-sided, with the exception of tests of heterogeneity and publication bias. Details of literature searches, study inclusion criteria, and meta-analysis are presented in the Supplementary Methods.

\section{Mendelian randomization Analysis}

The positive associations identified by the meta-analysis of cohort studies were further confirmed by twosample Mendelian randomization analysis. We searched for SLE in GWAS catalog (www.ebi.ac.uk/gwas) to identify the associations between SNPs and SLE, and the SNPs identified by the largest GWAS in populations of European ancestry or Asian ancestry were selected as instrumental variables respectively. We also calculated the proportion of variance $\left(R^{2}\right)$ explained in the risk factor by the SNP(s) and the strength of the instrument (F-statistic). Details of methodology for SNP selection, and calculation of the $\mathrm{R}^{2}$ and F-statistic are presented in the Supplementary Methods. We used the inverse variance weighted (IVW) fixed-effect method as the main method to estimate the effect of genetically predicted SLE on sitesspecific cancer in our Mendelian randomization analysis. The IVW method estimates the effect of the exposure on the outcome from the slope of the relationship between bXG (SNP-exposure association) and bYG (SNP-outcome association). In addition, other Mendelian randomization methods including MREgger, weighted median, and weighted mode method were used to check the consistency of the direction of effect estimates.

In sensitivity analyses, we assessed horizontal pleiotropy, heterogeneity tests, funnel plots, scatter plots and leave-one-out plots. In addition, scatter plots of effect estimates of individual SNPs with outcome versus effect estimates of individual SNPs with exposure are provided as a comparative visual assessment of the effect estimates generated from different Mendelian randomization methods. 
All analyses were conducted using the TwoSampleMR and MRInstruments R packages, curated by MRBase platform (www.mrbase.org). All tests were two-sided, and $P$ values of less than 0.05 were considered statistically significant unless stated otherwise.

\section{Results}

\section{Meta-analysis results}

The literature search and selection process was presented in Fig. 1. The comprehensive search generated 4292 potentially relevant studies, of which 4206 articles were excluded based on title and abstract review. We then examined full texts of the remaining 83 studies and excluded 39 of them. To be specific, 17 studies with irrelevant topics, 16 studies in which the study design was not cohort, four studies with incomplete data to calculate the estimates, and two studies being reviews were excluded. Additionally, four articles were included through previous review or reference lists of relevant studies. Finally, 48 eligible articles were included in the meta-analysis.

The characteristics of the included studies were summarized in Supplementary Table S1. 48 articles with a total of 247,575 patients with SLE (more than 178,332 females and 19,900 males) were represented. Among them, 43 studies estimated the cancer incidence in SLE patients with the follow-up period ranging from 1,000 person-years to 157,969 person-years or from 4.7 years to 35.3 years. Seven studies estimated cancer-specific death in SLE patients with the follow-up period ranging from 48 person-years to 91,669 person-years or from 8.1 years to 11.9 years. In addition, a total of 30 human cancers were systematically divided into six systemic groups (digestive cancers, respiratory cancers, reproductive cancers, urinary cancers, hematopoietic cancers, and other cancers).

The relationships between SLE and cancers were shown in Table 1 and Fig. 2. Our results suggested an increased cancer risk in SLE patients ( $R R=1.62,95 \% \mathrm{Cl}, 1.47-1.79)$. Site-specific analysis suggested that SLE were associated with an increased risk of lymphoma, Hodgkin's lymphoma, non-Hodgkin Lymphoma, leukemia, multiple myeloma, as well as esophagus, colon, anal, hepatobiliary, liver, pancreatic, larynx, lung, cervical, vagina/vulva, renal, bladder, skin (non-melanoma), and thyroid cancer. However, no significant associations were observed between SLE and cancers of stomach, colorectum, rectal, lip, oral cavity and pharynx, breast, ovary, uterus, prostate, melanoma, and brain.

The results of subgroup analysis by region were presented in Table 1 and Fig. 3. SLE patients were associated with an increased risk of overall cancers in Europe ( $\mathrm{RR}=1.66,95 \% \mathrm{Cl}, 1.35-2.03)$, America $(\mathrm{RR}=1.58,95 \% \mathrm{Cl}, 1.19-2.09)$, and Asia $(\mathrm{RR}=1.57,95 \% \mathrm{Cl}, 1.39-1.77)$. In region of Europe, we observed an increased risk of lymphoma, Hodgkin's lymphoma, non-Hodgkin Lymphoma, leukemia, as well as colon, anal, hepatobiliary, liver, pancreatic, larynx, lung, cervical, vagina/vulva, bladder, and skin (nonmelanoma) cancer. However, no significant associations were observed between SLE and cancers of esophagus, stomach, colorectum, rectal, lip, oral cavity and pharynx, breast, ovary, uterus, prostate, kidney, brain and thyroid, as well as Multiple myeloma and melanoma. In region of America, we observed an 
increased risk of liver cancer, lymphoma, Hodgkin's lymphoma and non-Hodgkin Lymphoma, whereas a deceased risk of prostate cancer in SLE patients. However, no significant associations were observed between SLE and cancers of colon, lung, breast, ovary, cervix, kidney, and melanoma. In region of Asia, SLE patients were associated with an increased risk of esophagus, hepatobiliary, liver, lip, oral cavity and pharynx, lung, cervical, skin (non-melanoma), brain and thyroid cancer, as well as lymphoma, nonHodgkin Lymphoma, and leukemia. However, no significant associations were observed between SLE and cancers of stomach, colorectum, pancreatic, breast, ovary, uterus, prostate, kidney, and bladder.

We also estimated the cancer-specific death in SLE patients, and the results were presented in Table 1 and Supplementary Figure S1. The cause-specific standard mortality ratio (SMR) in SLE patients was higher for overall cancer $(S M R=1.52,95 \% \mathrm{Cl}, 1.36-1.70)$, particularly for lung cancer $(S M R=1.52,95 \%$ $\mathrm{Cl}, 2.13-3.10)$. However, the cause-specific SMR for hematologic cancer was 1.42 (95\% $\mathrm{Cl}, 0.97-2.08)$.

\section{Mendelian randomization results}

The instrumental variable of SLE was constructed using 69 SNPs in European ancestry, explaining approximately $34 \%$ of the heritability totally, and the F-statistic was 172.36 (F > 100). In addition, 16 SNPs remained as the final genetic variants to construct instrumental variable of SLE in Asian ancestry, explaining over $1 \%$ of the variation, and the F-statistic was $10.47(F>10)$. The details of the SNPs enrolled in our study were represented in Supplementary Table S2 and Supplementary Table S3.

The significant associations ( 15 for SLE and sites-specific cancers in European ancestry, and 12 for SLE and sites-specific cancers in Asian ancestry) identified by the meta-analysis of cohort studies were further estimated by two-sample Mendelian randomization analysis. We searched for the outcome data using MR-Base and only found the data of colon cancer, pancreatic cancer, lung cancer, cervical cancer, bladder cancer, lymphoma, and Non-melanoma skin cancer in European ancestry, liver cancer and lung cancer in Asian ancestry. The details of outcome included in Mendelian randomization analyses were shown in Supplementary Table S4.

Table 2 presented associations of cancer risk in SLE patients as predicted using the instrument variants in Mendelian randomization analysis. Being consistent with the findings in the meta-analysis, the conventional IVW method indicated a causal association between genetically predisposed SLE and lymphoma $(\mathrm{OR}=1.0004,95 \% \mathrm{Cl}, 1.0001-1.0007, \mathrm{P}=0.0035)$, whereas a decreased risk of bladder cancer $(\mathrm{OR}=0.9996,95 \% \mathrm{Cl}, 0.9994-0.9998, \mathrm{P}=0.00004)$ in European ancestry. Furthermore, the MR-Egger and weighted median methods yielded similar results (Supplementary Table S5). However, no association was observed between genetically predisposed SLE and risk of colon, pancreatic, lung, cervical and Nonmelanoma skin cancer in European ancestry, liver cancer and lung cancer in Asian ancestry. Sensitivity analyses were performed to evaluate the robustness of the associations, and the results of horizontal pleiotropy and heterogeneity tests were shown in Supplementary Table S5.

Funnel plots, scatter plots and leave-one-out plots concerning relationship between SLE and lymphoma were shown in Supplementary figures S2-4. In addtion, leave-one-out analysis suggested that no single 
instrument was strongly driving the overall effect of SLE on other specific cancer types, indicating that these results were not sensitive to SNP selection (data not shown).

\section{Discussion}

Our meta-analysis included 48 cohort studies involving approximately 247,575 SLE patients to estimate the relationship between SLE and cancers. The present study demonstrated that SLE patients had a $62 \%$ elevated risk of overall cancer and a higher cause-specific SMR for overall cancer. In addition, our study yielded a significantly risk of site-specific cancers among SLE patients, especially esophagus, colon, anal, hepatobiliary, liver, pancreatic, larynx, lung, cervical, vagina/vulva, renal, bladder, skin (non-melanoma), and thyroid cancer, as well as lymphoma, Hodgkin's lymphoma, non-Hodgkin Lymphoma, leukemia, and multiple myeloma.

Furthermore, our study used 69 SNPs identified by the largest GWAS in European ancestry as the final genetic variants, which can explain over $34 \%$ of the heritability totally, suggesting a strong instrumental variable for analyzing the causal relation between SLE and disease risk. Using the Mendelian randomization approach, our study provided strong evidence for a possible causal association between SLE and lymphoma risk in European ancestry. As far as we know, our study provided the most comprehensive and latest evidence for assessing the causality between SLE and site-specific cancers risk through meta-analysis of cohort studies and Mendelian randomization analysis.

At present, the exact etiology underlying the attribution of SLE to cancer risk remains unclear. SLE is a chronic systemic autoimmune disease which could induce chronic multiorgan inflammatory lesion. The SLE-induced chronic inflammation may also promote the tissue injury, leading to the cancer development ${ }^{23}$. Furthermore, the chronic inflammation causing cellular apoptosis may also induce changes of cancerassociated genes ${ }^{24}$. On the other hand, immunosuppressive agents may also contribute to the onset of malignancies. It has been reported that immunosuppressive therapy, such as glucocorticoid and CTX, could also induce cytotoxic effects and suppress immune surveillance. Then the immune dysregulation of SLE may lead to the cellular damage and mutation, thus resulting in carcinogenesis of various cancers 25-28. Overall, more studies are warranted to further investigate the underlying mechanism.

According to our study, hematologic cancers, especially non-Hodgkin Lymphoma, is a common malignancy in SLE patients. Meta-analysis of cohort studies suggested that SLE patients had a 5.08-fold increased risk of lymphoma, 5.18-fold increased risk of non-Hodgkin Lymphoma, and 3.21-fold increased risk of Hodgkin's lymphoma. Moreover, both meta-analysis and Mendelian randomization analysis results revealed a potential causal relationship between SLE and lymphoma risk in European ancestry. Several potential mechanisms may account for the association between SLE and lymphoma. First, B cell and T cell activation play crucial roles in the pathogenesis of SLE and NHL, and the dysfunction of immune cells (such as B cell) in SLE patients may lead to abnormal B cell activation and proliferation, resulting in $B$ cell malignancies ${ }^{29}$. Second, chronic inflammation might heighten lymphoma risk in autoimmune diseases like SLE ${ }^{30}$. Third, SLE and lymphoma have similar genetic susceptibility or risk factors (e.g. 
Epstein-Barr virus infection) ${ }^{31-34}$. Fourth, the use of immunosuppressants (e.g. cyclophosphamide) might lead to lymphoma by direct mutagenesis or by disturbing immune surveillance 26,35 . Also, the animal models suggested an interaction between SLE and lymphoma ${ }^{36,37}$.

Interestingly, although meta-analysis results suggested an increased risk of bladder cancer, inconsistent results were observed in Mendelian randomization analysis. There are some plausible hypotheses that might explain the elevated risk of bladder cancers in patients with SLE. In particular, the use of immunosuppressive agents has an influence on the risk of bladder cancer in SLE patients. Several case series suggested that immunosuppressive agents increased the risk of bladder cancer in patients with SLE ${ }^{38-40}$. Otherwise, Bernasky et al. reported that SLE patients with immunosuppressive therapy had a $25 \%$ increased risk of bladder cancer ${ }^{41}$. However, these studies may have insufficient statistical power due to small sample sizes, and the development of SLE in relation to bladder cancer may be by chance. Therefore, we can hardly determine the relationship between SLE and bladder cancer risk according to the present evidence, and more related observational studies and biological studies are needed in the future.

The limitations of this study include: first, although we used the genetic variants identified by the largest GWAS as instrumental variable, it merely explained a part of variance of SLE. Especially in Asians, the instrumental variable of SLE could only explain approximately $1 \%$ of the variation. Second, we cannot estimate the relative contribution of genetic variants and environmental exposures to the association between SLE and cancer risk in our study, particularly because many additional genetic variants related to SLE have not yet been identified.

Third, results from the meta-analysis of cohort studies and Mendelian randomization analysis were inconsistent concerning the relationship between SLE and bladder cancer risk, so future well-designed cohort studies and Mendelian randomization analysis are needed to determine this association. Fourth, some outcome data were not found in MR-Base, therefore, we could hardly perform the Mendelian randomization analysis to identify the potentially causal relationships. Besides, some of the included studies in our meta-analysis were the retrospective studies, and the results might be confounded by recall bias and other factors.

\section{Conclusions}

In conclusion, our meta-analysis of cohort studies suggests that SLE patients are susceptible to overall cancers, particularly among Europeans. Besides, a higher risk of site-specific cancer of esophagus, colon, anal, hepatobiliary, liver, pancreatic, larynx, lung, cervical, vagina/vulva, renal, bladder, skin (nonmelanoma), and thyroid, as well as lymphoma, Hodgkin's lymphoma, non-Hodgkin Lymphoma, leukemia and multiple myeloma are observed among SLE patients. Furthermore, our study provides strong evidence that SLE is a risk factor for lymphoma and the association between them is likely to be causal based on data from a large meta-analysis of cohort studies and Mendelian randomization analysis. However, more well-designed cohort studies and Mendelian randomization analysis using more genetic variants are necessary to validate our findings. 


\section{Abbreviations}

$\mathrm{Cl}$

confidence intervals; GWAS, genome-wide association study; HR, hazard ratio; IVW, inverse variance weighted; MR, Mendelian randomization; OR, odds ratio; RR, relative risk; SIR, standard incidence ratio; SLE, systemic lupus erythematosus; SNP, single nucleotide polymorphisms.

\section{Article category}

Cancer epidemiology

\section{Declarations}

\section{Data availability}

The datasets analyzed and generated during the current study are available from the corresponding author on reasonable request. Systemic lupus erythematosus GWAS summary statistics are publicly available at https://www.ebi.ac.uk/gwas. Outcome summary statistics are publicly available at https://www.mrbase.org.

\section{Conflict of interest}

The authors declare that they have no conflict of interest.

\section{Acknowledgements}

This study was supported by the National Natural Science Foundation of China (81903393).

\section{Authors' contributions}

All authors contributed significantly to this work. B.Z. and D.G. designed the research study; D.G., M.T., and M.Z. collected the data; H.C., S.C., Y.W., Y.B., Z.Z, Y.T., and X.W. analyzed the data; D.G. wrote the first draft of the manuscript. All authors reviewed, edited and approved the manuscript.

\section{References}

1. Durcan L, O'Dwyer T, Petri M. Management strategies and future directions for systemic lupus erythematosus in adults. Lancet 2019;393:2332-2343.

2. Lim SS, Drenkard C, McCune WJ, et al. Population-based lupus registries: advancing our epidemiologic understanding. Arthritis Rheum 2009;61:1462-6.

3. Bertsias G, loannidis JP, Boletis J, et al. EULAR recommendations for the management of systemic lupus erythematosus. Report of a Task Force of the EULAR Standing Committee for International Clinical Studies Including Therapeutics. Ann Rheum Dis 2008;67:195-205.

4. Tsokos GC. Systemic lupus erythematosus. N Engl J Med 2011;365:2110-21. 
5. Wang LH, Wang WM, Lin SH, et al. Bidirectional relationship between systemic lupus erythematosus and non-Hodgkin's lymphoma: a nationwide population-based study. Rheumatology (Oxford) 2019;58:1245-1249.

6. Bae EH, Lim SY, Han KD, et al. Systemic lupus erythematosus is a risk factor for cancer: a nationwide population-based study in Korea. Lupus 2019;28:317-323.

7. Kuo CF, Chou IJ, Rees F, et al. Temporal relationships between systemic lupus erythematosus and comorbidities. Rheumatology (Oxford) 2019;58:840-848.

8. Tallbacka KR, Pettersson T, Pukkala E. Increased incidence of cancer in systemic lupus erythematosus: a Finnish cohort study with more than 25 years of follow-up. Scand J Rheumatol 2018;47:461-464.

9. Ramsey-Goldman R, Brar A, Richardson C, et al. Standardised incidence ratios (SIRs) for cancer after renal transplant in systemic lupus erythematosus (SLE) and non-SLE recipients. Lupus Sci Med 2016;3:e000156.

10. Hemminki K, Liu X, Ji J, et al. Effect of autoimmune diseases on risk and survival in histologyspecific lung cancer. Eur Respir J 2012;40:1489-95.

11. Dey D, Kenu E, Isenberg DA. Cancer complicating systemic lupus erythematosus-a dichotomy emerging from a nested case-control study. Lupus 2013;22:919-27.

12. Yu KH, Kuo CF, Huang LH, et al. Cancer Risk in Patients With Inflammatory Systemic Autoimmune Rheumatic Diseases: A Nationwide Population-Based Dynamic Cohort Study in Taiwan. Medicine (Baltimore) 2016;95:e3540.

13. Parikh-Patel A, White RH, Allen M, et al. Cancer risk in a cohort of patients with systemic lupus erythematosus (SLE) in California. Cancer Causes Control 2008;19:887-94.

14. Lawlor DA, Davey Smith G, Kundu D, et al. Those confounded vitamins: what can we learn from the differences between observational versus randomised trial evidence? Lancet 2004;363:1724-7.

15. Davey Smith G, Ebrahim S. Epidemiology-is it time to call it a day? Int J Epidemiol 2001;30:1-11.

16. Smith GD, Ebrahim S. 'Mendelian randomization': can genetic epidemiology contribute to understanding environmental determinants of disease? Int J Epidemiol 2003;32:1-22.

17. Davey Smith G, Hemani G. Mendelian randomization: genetic anchors for causal inference in epidemiological studies. Hum Mol Genet 2014;23:R89-98.

18. Pierce BL, Burgess S. Efficient design for Mendelian randomization studies: subsample and 2-sample instrumental variable estimators. Am J Epidemiol 2013;178:1177-84.

19. Yarmolinsky J, Wade KH, Richmond RC, et al. Causal Inference in Cancer Epidemiology: What Is the Role of Mendelian Randomization? Cancer Epidemiol Biomarkers Prev 2018;27:995-1010.

20. Bentham J, Morris DL, Graham DSC, et al. Genetic association analyses implicate aberrant regulation of innate and adaptive immunity genes in the pathogenesis of systemic lupus erythematosus. Nat Genet 2015;47:1457-1464. 
21. Peng H, Li C, Wu X, et al. Association between systemic lupus erythematosus and lung cancer: results from a pool of cohort studies and Mendelian randomization analysis. J Thorac Dis 2020;12:5299-5302.

22. Moher D, Liberati A, Tetzlaff J, et al. Preferred reporting items for systematic reviews and metaanalyses: the PRISMA statement. BMJ 2009;339:b2535.

23. Enzler T, Gillessen S, Manis JP, et al. Deficiencies of GM-CSF and interferon gamma link inflammation and cancer. J Exp Med 2003;197:1213-9.

24. Itzkowitz SH, Yio X. Inflammation and cancer IV. Colorectal cancer in inflammatory bowel disease: the role of inflammation. Am J Physiol Gastrointest Liver Physiol 2004;287:G7-17.

25. Drevet G, Duruisseaux M, Maury JM, et al. Lung cancer surgical treatment after solid organ transplantation: A single center 30-year experience. Lung Cancer 2020;139:55-59.

26. Kang KY, Kim HO, Yoon HS, et al. Incidence of cancer among female patients with systemic lupus erythematosus in Korea. Clin Rheumatol 2010;29:381-8.

27. Chun BC, Bae SC. Mortality and cancer incidence in Korean patients with systemic lupus erythematosus: results from the Hanyang lupus cohort in Seoul, Korea. Lupus 2005;14:635-8.

28. Talar-Williams C, Hijazi YM, Walther MM, et al. Cyclophosphamide-induced cystitis and bladder cancer in patients with Wegener granulomatosis. Ann Intern Med 1996;124:477-84.

29. Azevedo PC, Murphy G, Isenberg DA. Pathology of systemic lupus erythematosus: the challenges ahead. Methods Mol Biol 2014;1134:1-16.

30. Dias C, Isenberg DA. Susceptibility of patients with rheumatic diseases to B-cell non-Hodgkin lymphoma. Nat Rev Rheumatol 2011;7:360-8.

31. Din L, Sheikh M, Kosaraju N, et al. Genetic overlap between autoimmune diseases and non-Hodgkin lymphoma subtypes. Genet Epidemiol 2019;43:844-863.

32. Baecklund E, Smedby KE, Sutton LA, et al. Lymphoma development in patients with autoimmune and inflammatory disorders-what are the driving forces? Semin Cancer Biol 2014;24:61-70.

33. Tarr T, Gyorfy B, Szekanecz E, et al. Occurrence of malignancies in Hungarian patients with systemic lupus erythematosus: results from a single center. Ann N Y Acad Sci 2007;1108:76-82.

34. Abu-Shakra M, Ehrenfeld M, Shoenfeld Y. Systemic lupus erythematosus and cancer: associated or not? Lupus 2002;11:137-44.

35. Szekanecz Z, Szekanecz E, Bako G, et al. Malignancies in autoimmune rheumatic diseases - a minireview. Gerontology 2011;57:3-10.

36. Haabeth OA, Lorvik KB, Hammarstrom C, et al. Inflammation driven by tumour-specific Th1 cells protects against B-cell cancer. Nat Commun 2011;2:240.

37. Sugai S, Palmer DW, Talal N, et al. Protective and cellular immune responses to idiotypic determinants on cells from a spontaneous lymphoma of NZB-NZW F1 mice. J Exp Med 1974;140:1547-58. 
38. Chow SK, Looi LM, Loh CS, et al. Cyclophosphamide-induced transitional cell carcinoma of bladder in lupus nephritis. Intern Med J 2002;32:114-6.

39. Ortiz A, Gonzalez-Parra E, Alvarez-Costa G, et al. Bladder cancer after cyclophosphamide therapy for lupus nephritis. Nephron 1992;60:378-9.

40. Thrasher JB, Miller GJ, Wettlaufer JN. Bladder leiomyosarcoma following cyclophosphamide therapy for lupus nephritis. J Urol 1990;143:119-21.

41. Bernatsky S, Joseph L, Boivin JF, et al. The relationship between cancer and medication exposures in systemic lupus erythaematosus: a case-cohort study. Ann Rheum Dis 2008;67:74-9.

\section{Figures}

\section{Figure 1 Flow diagram of literature search and study selection}

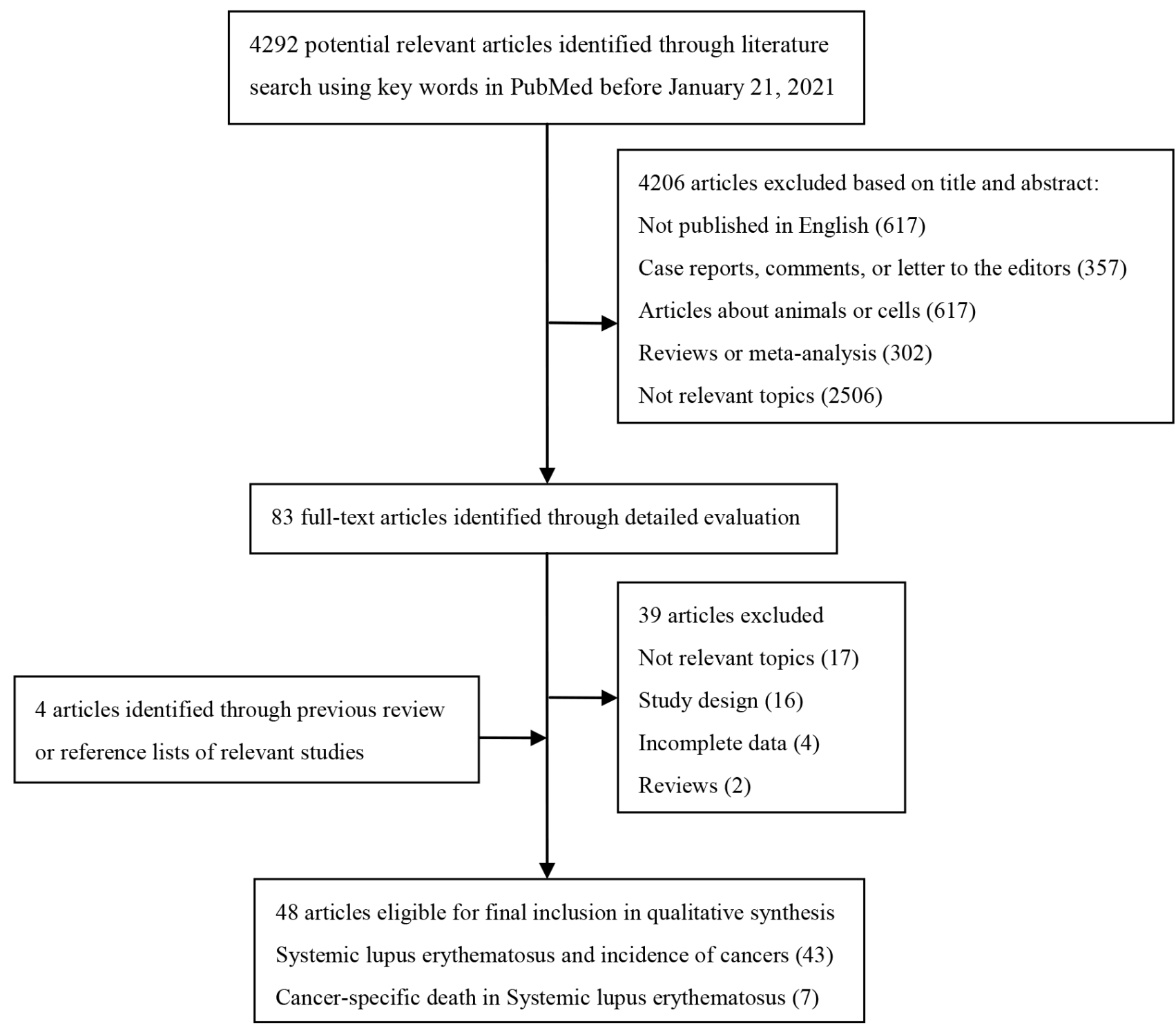


Figure 1. Flow diagram of literature search and study selection.

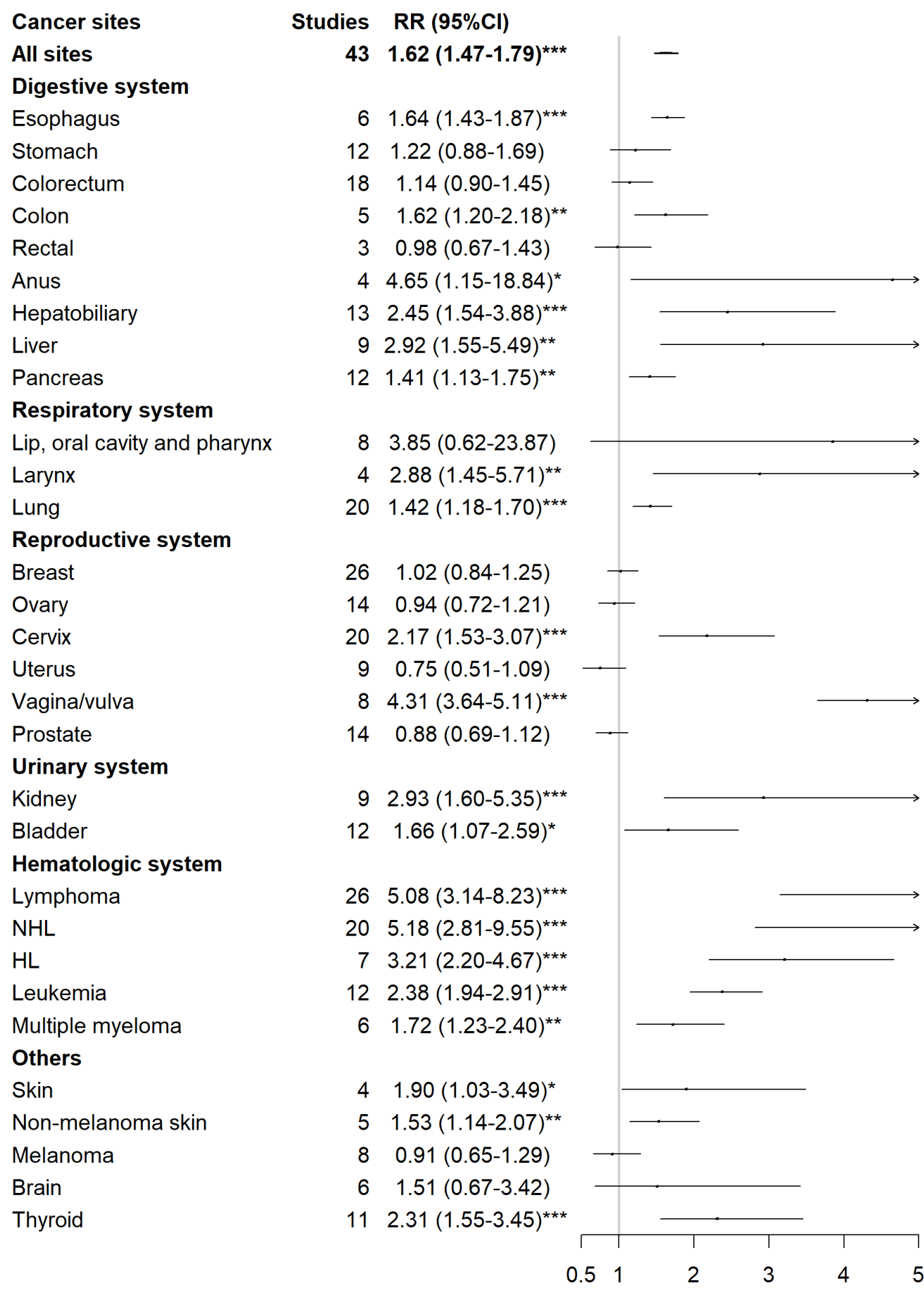

Figure 2

Figure 2. Overall cancer and site-specific cancer risk in patients with systemic lupus erythematosus based on meta-analysis of cohort studies. 

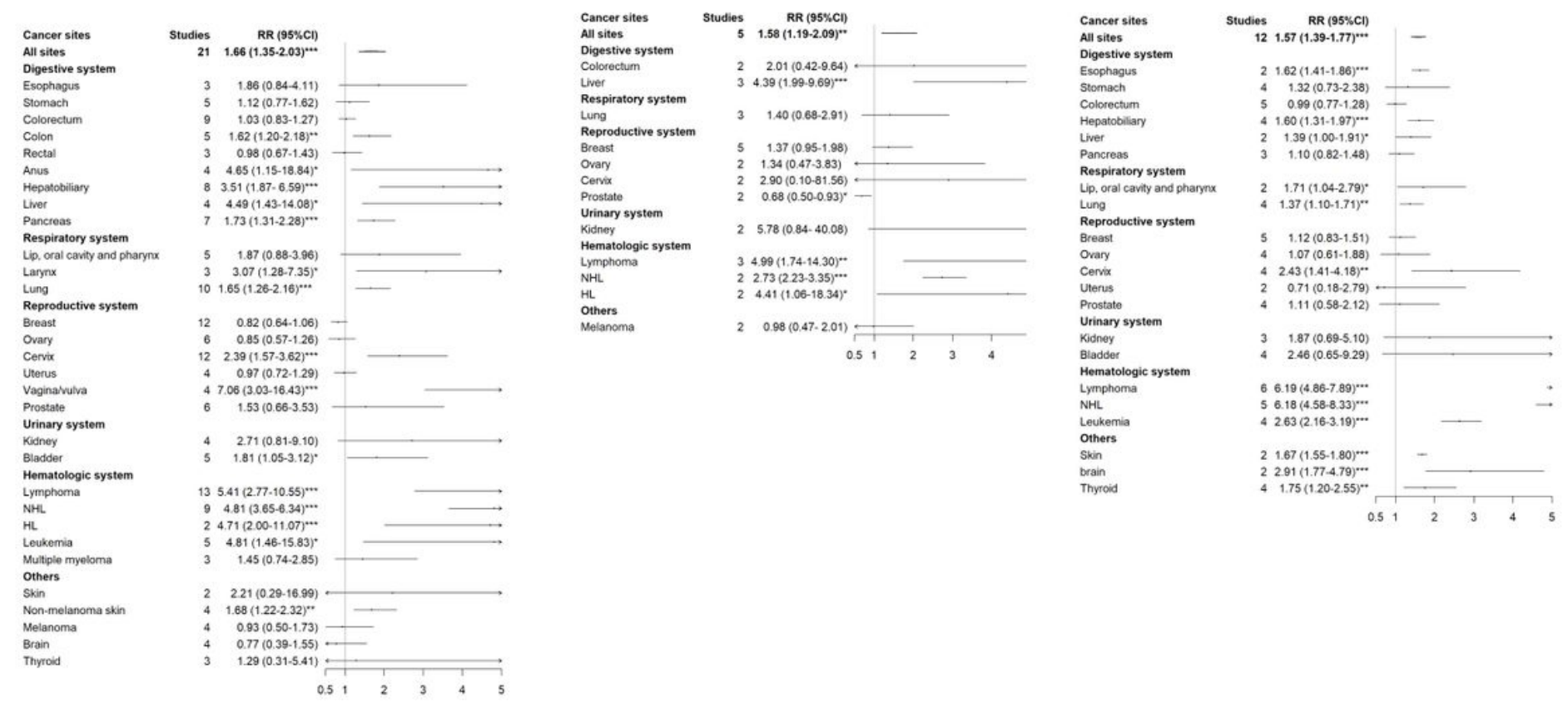

Figure 3

Overall cancer and site-specific cancer risk in patients with systemic lupus erythematosus based on metaanalysis of cohort studies in different region (A. Europe, B. America, C. Asia)

\section{Supplementary Files}

This is a list of supplementary files associated with this preprint. Click to download.

- Supplementarymaterial.docx 